\title{
Use Hierarchical Codebook to Improve the Primary User Detection in the Cognitive Radio's Cooperative Spectrum Sensing
}

\author{
Hoai Trung Tran
}

\begin{abstract}
Currently, the cognitive network is receiving much attention due to the advantages it brings to users. An important method in cognitive radio networks is spectrum sensing, as it allows secondary users (SUs) to detect the existence of a primary user (PU). Information of probability of false detection or warning about the $\mathrm{PU}$ is sent to a fusion center (FC) by the SUs, from which the FC will decide whether or not to allow the SUs to use the PU spectrum to obtain information. The transmission of information with a high signal to noise ratio (SNR) will increase the FC's ability to detect the existence of the PU. However, researchers are currently focusing on probabilistic formulas assuming that the channel is known ideally or there is nominal channel information at the FC; moreover, one model where the FC only knows the channel correlation matrix. Furthermore, studies are still assuming this is a simple multiple input - multiple output (MIMO) channel model but do not pay much attention to the signal processing at the transmitting and receiving antennas between the SUs and the FCs. A new method introduced in this paper when combining beamforming and hierarchical codebook makes the ability to detect the existence of the PU at the FC significantly increased compared to traditional methods.
\end{abstract}

Keywords - Beamforming, Cooperative spectrum sensing, Hierarchical codebook, Generalized likelihood ratio test.

\section{INTRODUCTION}

Currently, the cognitive radio network is proposed to solve the spectrum scarcity problem due to spectrum occupation variation [1], [2], and the bandwidths below $6 \mathrm{GHz}$ are heavily used [1]. Using the suitable technique for the cognitive radio will increase the efficiency of the network. Currently, contributions are focused on various configurations of cooperative cognitive systems to increase the speed of SUs in the presence or absence of PUs [3]-[11].

The popular technique used for cognition commonly used today is spectrum sensing (SS), which finds the white spaces in the spectrum. In this technique, the SUs needs to detect the signal of the PU [1], [2]. Because of the effects of the propagating atmospheres, the $\mathrm{PU}$ signal is not sensed by the SU unambiguously [1]. Determining whether or not PUs is present is very important for an underlay and overlay system, especially the overlay case. The concept of cooperation is understood in a different sense when the SUs cooperates to find out the presence of the PUs and send it to the Fusion center $(\mathrm{FC})$ to decide whether these PUs are present or not. There the concept of fusion center is introduced in which the combination of signals from the SUs to the FC to make the decision whether the PU is present or not and thereby allow the SUs to operate or not [1]. The system that uses SUs combined in this fashion is called the cooperative spectrum sensing (CSS) system.

The MIMO role should be taken care of in this CSS system. Meanwhile, the PU recognition algorithm is interested to save time and energy through the two-stage algorithm, implemented from the SUs to the PU [2]. If SNR is high, the algorithm needs only one stage. Energy and speed are considered in [12] when cooperative spectrum sensing at the FC from the cooperating SUs using 1 bit report. In [13], applied MIMO to a cooperative system was introduced in which the reporting channels from the SUs to the FC were Rayleigh fading channels with additive white Gaussian noise. The use of MIMO in fading environment is entirely suitable for the CR systems due to spatial diversity, so it has better detection quality [13]. The signal acquisition method is also implemented in different ways, such as equal gain combiner (EGC), maximum ratio combiner (MRC), and optimum combiner (OC), to improve the PU detection at the FC.

The term generalized likelihood ratio test (GLRT) is appearing in [1], [14], [15]. This algorithm uses the statistic test that describes the probability of assuming the presence and absence of the PU using threshold level for decision. If this ratio is greater than the threshold level, the $\mathrm{PU}$ is present and vice versa. Two characteristic quantities, detection probability and false alarm probability are considered to be two important parameters assessing the ability to determine the presence of the PU to what extent. These parameters are related by the receiver operating characteristic (ROC) curve, where the vertical axis is the detection probability, and the horizontal axis is the false alarm probability. The greater slope of the ROC, the better the ability to detect the actual existence of the PU. Meanwhile, the less the ability to see the PU even though the PU does not exist (false alarm) [1], [15], [16]. The CSS system included MIMO where the SUs transmit local hard decisions to the FC through multiple antennas and the $\mathrm{FC}$ receives the signal through multiple antennas [1]; this increases the FC's spectrum sensing ability of the PU, and then its decision will be much better. The concept of MIMO CSS has since been considered [1]. The FC uses the GLRT performed in which the unknown noise correlation matrix with CSI is imperfect and perfect at the SUs [14]. Approaches for signal acquisition from STs are 
given in [15], where sensing systems are based on the likelihood ratio test (LRT) optimally assuming that perfect CSI between the SU and the FC. Next, the LRT algorithm for uncertainty statistics ratio test and the robust generalized likelihood ratio test (R-GLRT) algorithm for CSI uncertainty are determined for cooperative spectrum sensing environments.

To increase the efficiency of using MIMO leading to increased detection and false alarm probabilities for the GLRT algorithm at the receiver, some spatial processing is needed for the receive antennas at the FC. The selection of antenna numbers at the FC is dialed [1], which is an interesting topic. The use of the antenna at the FC depends on the presence of the PU. If the PU exists and the FC sense is passed through cooperative SUs, the number of antennas is reduced to ensure that the interference from the $\mathrm{FC}$ to the $\mathrm{PU}$ is not much. If no PU is present, the FC will make maximum use of the antennas for transmission. However, it is not interested in the beamforming technique since this technique helps increase the signal-to-noise ratio, or in other words, it increases the decision capability at the FC. This solution was studied only in [16]; however, beamforming is applied to a single angle to the Angle of Arrival (AoA), at the simple level, not to some physical transmission paths as in multipath environments. Furthermore, [16] is only interested in determining the presence of the PU at a single SU, which is not as complex as compiling reporting signals from many SUs, then the FC deciding whether or not the presence of the PU. In other hand, some papers use hierarchical codebook to improve the success rate [17] using broadening the beamwidth of antenna subarrays and deactivations, the capacity [18] and Doppler frequency offset compensation [19] by finding relative the Angle of Departure (AoD) and the AoA pair, however they do not consider how to solve problem of knowing the presence of the PU [19], [20]. This paper uses the beamforming technique combined with the hierarchical codebook, first to find the pairs of AoD and AoA corresponding to the physical paths, then using the test parameters to determine it is better than using the SVD of a channel correlation matrix (TR - Robust Test) or nominal CSI information (TUA - Uncertainty Agnostic Test) [15] at the SUs when reporting to the FC.

\section{CHANNEL MODEL}

The channel model can be described, specifically at the time at the $\mathrm{FC}$, as below:

$$
\mathbf{y}_{\mathrm{i}}(\mathrm{l})=\mathbf{H}_{\mathrm{i}} \mathbf{u}_{\mathrm{i}}(\mathrm{l})+\mathbf{w}_{\mathrm{i}}(\mathrm{l})
$$

$\mathbf{u}_{\mathrm{i}}(\mathrm{l})$ is the transmit signal from the ith SU at the time of 1 , having size of $\mathrm{N}_{\mathrm{c}} \times 1$. This $\mathbf{U}_{\mathrm{i}}=\left[\begin{array}{llll}\mathbf{u}_{\mathrm{i}}(1) & \mathbf{u}_{\mathrm{i}}(2) & \ldots & \mathbf{u}_{\mathrm{i}}(\mathrm{L})\end{array}\right]$ represents the decision of the ith SU about the presence or in presence of the PU after the L times of observation.

$\mathbf{H}_{\mathrm{i}}$ is the channel matrix from the ith $\mathrm{SU}$ to the $\mathrm{FC}$ at the time of 1 , having size of $\mathrm{N}_{\mathrm{f}} \times \mathrm{N}_{\mathrm{c}}$. Suppose we have a channel model with a transmitter and a receiver, plus relays in between them. Source, each of the relays, and receiver are all equipped with multiple antennas to transmit and receive data.

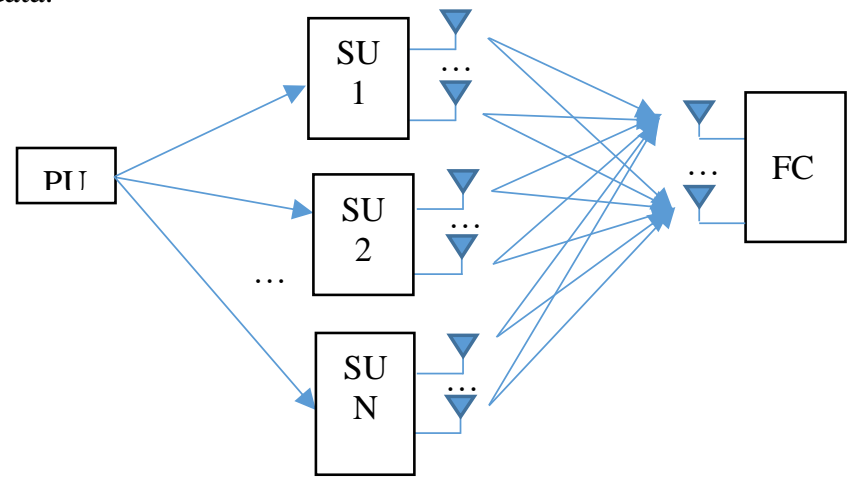

Fig. 1. Channel model consisting of $K$ relays.

$$
\mathbf{H}_{\mathrm{i}}=\left[\begin{array}{cccc}
\mathrm{h}_{\mathrm{i}, 1}(1) & \mathrm{h}_{\mathrm{i}, 2}(1) & \ldots & \mathrm{h}_{\mathrm{i}, \mathrm{N}_{\mathrm{f}}}(1) \\
\mathrm{h}_{\mathrm{i}, 1}(2) & \mathrm{h}_{\mathrm{i}, 2}(2) & \ldots & \mathrm{h}_{\mathrm{i}, \mathrm{N}_{\mathrm{f}}}(2) \\
\ldots & \ldots & \ldots & \ldots \\
\mathrm{h}_{\mathrm{i}, 1}\left(\mathrm{~N}_{\mathrm{c}}\right) & \mathrm{h}_{\mathrm{i}, 2}\left(\mathrm{~N}_{\mathrm{c}}\right) & \ldots & \mathrm{h}_{\mathrm{i}, \mathrm{N}_{\mathrm{f}}}\left(\mathrm{N}_{\mathrm{c}}\right)
\end{array}\right]^{\mathrm{H}}
$$

$\mathbf{w}_{\mathrm{i}}(1)$ is the noise vector received at the FC that interferes at the received signal $\mathrm{y}_{\mathrm{i}}(\mathrm{l})$, having size of $\mathrm{N}_{\mathrm{f}} \times 1$.

The received signal at the jth antenna of the FC:

$$
\begin{aligned}
& \mathrm{y}_{\mathrm{i}, \mathrm{j}}(1)=\mathbf{h}_{\mathrm{i}, \mathrm{j}}^{\mathrm{H}} \mathbf{u}_{\mathrm{i}}(1)+\mathrm{w}_{\mathrm{i}, \mathrm{j}}(\mathrm{l}) \\
& \mathbf{y}_{\mathrm{i}}(1)=\left[\begin{array}{llll}
\mathrm{y}_{\mathrm{i}, 1}(1) & \mathrm{y}_{\mathrm{i}, 2}(1) & . . & \mathrm{y}_{\mathrm{i}, \mathrm{N}_{\mathrm{f}}}(1)
\end{array}\right]^{\mathrm{H}} \\
& \mathbf{w}_{\mathrm{i}}(1)=\left[\begin{array}{llll}
\mathrm{w}_{\mathrm{i}, 1}(1) & \mathrm{w}_{\mathrm{i}, 2}(1) & . . & \mathrm{w}_{\mathrm{i}, \mathrm{N}_{\mathrm{f}}}(1)
\end{array}\right]^{\mathrm{H}} \\
& \mathbf{H}_{\mathrm{i}}=\left[\begin{array}{llll}
\mathbf{h}_{\mathrm{i}, 1} & \mathbf{h}_{\mathrm{i}, 2} & . . & \mathbf{h}_{\mathrm{i}, \mathrm{N}_{\mathrm{f}}}
\end{array}\right]^{\mathrm{H}}
\end{aligned}
$$

where $\mathbf{h}_{\mathrm{i}, \mathrm{j}}$ has size of $\mathrm{N}_{\mathrm{c}} \times 1$

If we combine the $\mathrm{L}$ transmit vectors, we have the signal at the jth antenna of the FC:

$$
\mathbf{y}_{\mathrm{i}, \mathrm{j}}=\mathbf{U}_{\mathrm{i}} \mathbf{h}_{\mathrm{i}, \mathrm{j}}+\mathbf{w}_{\mathrm{i}, \mathrm{j}}
$$

where

$$
\begin{aligned}
& \mathbf{y}_{i, j}=\left[\begin{array}{llll}
y_{i, j}(1) & y_{i, j}(2) & \ldots & y_{i, j}(L)
\end{array}\right]^{H} \\
& \mathbf{w}_{i, j}=\left[\begin{array}{llll}
w_{i, j}(1) & w_{i, j}(2) & . . & w_{i, j}(L)
\end{array}\right]^{H} \\
& \mathbf{U}_{i}=\left[\begin{array}{lllll}
\mathbf{u}_{i}(1) & \mathbf{u}_{i}(2) & . . & \mathbf{u}_{i}(L)
\end{array}\right]^{\mathrm{H}} \\
& =\left[\begin{array}{cccc}
u_{i, 1}(1) & u_{i, 2}(2) & \ldots & u_{i, L}\left(N_{c}\right) \\
u_{i, 2}(1) & u_{i, 2}(2) & \ldots & u_{i, L}\left(N_{c}\right) \\
\ldots & \ldots & \ldots & \ldots \\
u_{i, N_{c}}(1) & u_{i, N_{c}}(2) & \ldots & u_{i, L}\left(N_{c}\right)
\end{array}\right]^{H}
\end{aligned}
$$

has size of $\mathrm{L} \times \mathrm{N}_{\mathrm{c}}$

$\mathbf{U}_{\mathrm{i}}=\mathbf{P}_{1}$ is assumed as in case of the presence of PU, and $\mathbf{U}_{\mathrm{i}}=\mathbf{P}_{0}$ when absent of PU.

Noise matrix: 


$$
\begin{aligned}
& \mathbf{W}_{\mathrm{i}}=\left[\begin{array}{lccc}
\mathbf{w}_{\mathrm{i}}(1) & \mathbf{w}_{\mathrm{i}}(2) & \ldots & \mathbf{w}_{\mathrm{i}}(\mathrm{L})
\end{array}\right] \\
& =\left[\begin{array}{cccc}
\mathrm{w}_{\mathrm{i}, 1}(1)^{*} & \mathrm{w}_{\mathrm{i}, 2}(2)^{*} & \ldots & \mathrm{u}_{\mathrm{i}, 1}(\mathrm{~L})^{*} \\
\mathrm{w}_{\mathrm{i}, 2}(1)^{*} & \mathrm{w}_{\mathrm{i}, 2}(2)^{*} & \ldots & \mathrm{u}_{\mathrm{i}, 2}(\mathrm{~L})^{*} \\
\ldots & \ldots & \ldots & \ldots \\
\mathrm{w}_{\mathrm{i}, \mathrm{N}_{\mathrm{f}}}(1)^{*} & \mathrm{u}_{\mathrm{i}, \mathrm{N}_{\mathrm{f}}}(2)^{*} & \ldots & \mathrm{u}_{\mathrm{i}, \mathrm{N}_{\mathrm{f}}}(\mathrm{L})^{*}
\end{array}\right]=\left[\begin{array}{c}
\mathbf{w}_{\mathrm{i}, 1}^{\mathrm{T}} \\
\mathbf{w}_{\mathrm{i}, 2}^{\mathrm{T}} \\
\ldots \\
\mathbf{w}_{\mathrm{i}, \mathrm{N}_{\mathrm{f}}}^{\mathrm{T}}
\end{array}\right]
\end{aligned}
$$

Based on the generalized formula of the probability of detection and false alarm, we have:

$$
\begin{aligned}
& \mathrm{P}_{\mathrm{D}}=\operatorname{Prob}\left\{\mathrm{T}(\mathbf{y})>\gamma \mid \mathrm{H}_{1} \text { true }\right\}=\int_{\gamma}^{\infty} \mathrm{p}\left(\mathbf{y}, \mathrm{H}_{1}\right) \mathrm{dT}(\mathbf{y}) \\
& \mathrm{P}_{\mathrm{FA}}=\operatorname{Prob}\left\{\mathrm{T}(\mathbf{y})>\gamma \mid \mathrm{H}_{0} \text { true }\right\}=\int_{\gamma}^{\infty} \mathrm{p}\left(\mathbf{y}, \mathrm{H}_{0}\right) \mathrm{dT}(\mathbf{y})
\end{aligned}
$$

Test parameter is based on Neyman - Pearson lemma; the aim is to increase the probability of detection while the probability of false alarm keeps constant. Therefore, $\mathrm{T}(\mathrm{y})$ can be ratio between the probability of the signal $\mathbf{y}$ as $\mathrm{H}_{1}$ known and probability of signal $\mathbf{y}$ as $\mathrm{H}_{0}$ known:

$$
\mathrm{T}(\mathrm{y})=\ln \left[\frac{\mathrm{p}\left(\mathbf{y}, \mathrm{H}_{1}\right)}{\mathrm{p}\left(\mathbf{y}, \mathrm{H}_{0}\right)}\right]_{\mathrm{H}_{0}}^{\mathrm{H}_{1}} \gamma
$$

Serving (8) with:

$$
\begin{aligned}
& P\left(\mathbf{y}_{i}, \mathbf{U}_{i}=\mathbf{P}_{1}\right)=\prod_{j=1}^{N_{f}} \frac{1}{\pi^{L} \sigma^{2 L}} \exp \left[-\frac{1}{\sigma^{2}}\left(\mathbf{y}_{i, j}-P_{1} \mathbf{h}_{i, j}\right)^{H}\right. \\
& \left.\mathbf{I}_{\mathrm{L}}^{-1}\left(\mathbf{y}_{\mathrm{i}, \mathrm{j}}-\mathrm{P}_{1} \mathbf{h}_{\mathrm{i}, \mathrm{j}}\right)\right] \\
& =\frac{1}{\pi^{\mathrm{L}} \sigma^{2 \mathrm{~L}}} \exp \left(-\sum_{\mathrm{j}=1}^{\mathrm{N}_{\mathrm{f}}} \frac{\left\|\mathbf{y}_{\mathrm{i}, \mathrm{j}}-\mathbf{P}_{1} \mathbf{h}_{\mathrm{i}, \mathrm{j}}\right\|^{2}}{\sigma^{2}}\right) \\
& P\left(\mathbf{y}_{i}, \mathbf{U}_{i}=\mathbf{P}_{0}\right)=\prod_{j=1}^{N_{f}} \frac{1}{\pi^{L} \sigma^{2 L}} \exp \left[-\frac{1}{\sigma^{2}}\left(\mathbf{y}_{i, j}-P_{0} \mathbf{h}_{i, j}\right)^{H}\right. \\
& \left.\mathbf{I}_{\mathrm{L}}^{-1}\left(\mathbf{y}_{\mathrm{i}, \mathrm{j}}-\mathrm{P}_{0} \mathbf{h}_{\mathrm{i}, \mathrm{j}}\right)\right] \\
& =\frac{1}{\pi^{\mathrm{L}} \sigma^{2 \mathrm{~L}}} \exp \left(-\sum_{\mathrm{j}=1}^{\mathrm{N}_{\mathrm{f}}} \frac{\left\|\mathbf{y}_{\mathrm{i}, \mathrm{j}}-\mathbf{P}_{0} \mathbf{h}_{\mathrm{i}, \mathrm{j}}\right\|^{2}}{\sigma^{2}}\right) \\
& \mathrm{P}_{\mathrm{r}}\left(\mathbf{U}_{\mathrm{i}}=\mathbf{P}_{1} \mid \mathrm{H}_{1}\right)=\mathrm{P}_{\mathrm{D}, \mathrm{i}} \text { and } \mathrm{P}_{\mathrm{r}}\left(\mathbf{U}_{\mathrm{i}}=\mathbf{P}_{1} \mid \mathrm{H}_{0}\right)=\mathrm{P}_{\mathrm{F}, \mathrm{i}} \\
& \mathrm{T}(\mathrm{y})=\sum_{\mathrm{i}=1}^{\mathrm{N}} \ln \left[\frac{\mathrm{P}\left(\mathbf{y}_{\mathrm{i}} ; \mathbf{U}_{\mathrm{i}}=\mathbf{P}_{1}\right) \mathrm{P}_{\mathrm{r}}\left(\mathbf{U}_{\mathrm{i}}=\mathbf{P}_{1} \mid \mathrm{H}_{1}\right)+\mathrm{P}\left(\mathbf{y}_{\mathrm{i}} ; \mathbf{U}_{\mathrm{i}}=\mathbf{P}_{0}\right) \mathrm{P}_{\mathrm{r}}\left(\mathbf{U}_{\mathrm{i}}=\mathbf{P}_{0} \mid \mathrm{H}_{1}\right)}{\mathrm{P}\left(\mathbf{y}_{\mathrm{i}} ; \mathbf{U}_{\mathrm{i}}=\mathbf{P}_{1}\right) \mathrm{P}_{\mathrm{r}}\left(\mathbf{U}_{\mathrm{i}}=\mathbf{P}_{1} \mid \mathrm{H}_{0}\right)+\mathrm{P}\left(\mathbf{y}_{\mathrm{i}} ; \mathbf{U}_{\mathrm{i}}=\mathbf{P}_{0}\right) \mathrm{P}_{\mathrm{r}}\left(\mathbf{U}_{\mathrm{i}}=\mathbf{P}_{0} \mid \mathrm{H}_{0}\right)}\right] \\
& =\sum_{i=1}^{N} \ln \left[\frac{P_{D, i} \exp \left(-\sum_{j=1}^{N_{f}} \frac{\left\|\mathbf{y}_{i, j}-\mathbf{P}_{1} \mathbf{h}_{i, j}\right\|^{2}}{\sigma^{2}}\right)+\left(1-P_{D, i}\right) \exp \left(-\sum_{j=1}^{N_{f}} \frac{\left\|\mathbf{y}_{i, j}-\mathbf{P}_{0} \mathbf{h}_{i, j}\right\|^{2}}{\sigma^{2}}\right)}{P_{F, i} \exp \left(-\sum_{j=1}^{N_{f}} \frac{\left\|\mathbf{y}_{i, j}-\mathbf{P}_{1} \mathbf{h}_{\mathrm{i}, j}\right\|^{2}}{\sigma^{2}}\right)+\left(1-P_{F, i}\right) \exp \left(-\sum_{j=1}^{N_{f}} \frac{\left\|\mathbf{y}_{i, j}-\mathbf{P}_{0} \mathbf{h}_{i, j}\right\|^{2}}{\sigma^{2}}\right)}\right]
\end{aligned}
$$

Specific case:

If $\mathbf{P}_{1}=-\mathbf{P}_{0}=\mathbf{P}$ we have:

$$
\mathrm{T}(\mathrm{y})=\sum_{\mathrm{i}=1}^{N} \ln \left[\frac{\mathrm{P}_{\mathrm{D}, \mathrm{i}}+\left(1-\mathrm{P}_{\mathrm{D}, \mathrm{i}}\right) \exp \left(-\frac{4}{\sigma^{2}} \sum_{\mathrm{j}=1}^{\mathrm{N}_{\mathrm{f}}} \operatorname{Re}\left(\mathbf{y}_{\mathrm{i}, \mathrm{j}}^{\mathrm{H}} \mathbf{P h}_{\mathrm{i}, \mathrm{j}}\right)\right)}{\mathrm{P}_{\mathrm{F}, \mathrm{i}}+\left(1-\mathrm{P}_{\mathrm{F}, \mathrm{i}}\right) \exp \left(-\frac{4}{\sigma^{2}} \sum_{\mathrm{j}=1}^{\mathrm{N}_{\mathrm{f}}} \operatorname{Re}\left(\mathbf{y}_{\mathrm{i}, \mathrm{j}}^{\mathrm{H}} \mathbf{P h}_{\mathrm{i}, \mathrm{j}}\right)\right)}\right]
$$

Reduced more to assume that $\exp (-\mathrm{t})=1-\mathrm{t}$ and $\ln (1+\mathrm{t})=\mathrm{t}$, finally we have:

$$
\mathrm{T}(\mathrm{y})=\sum_{\mathrm{i}=1}^{\mathrm{N}} \mathrm{a}_{\mathrm{i}} \sum_{\mathrm{j}=1}^{\mathrm{N}_{\mathrm{f}}} \operatorname{Re}\left(\mathbf{y}_{\mathrm{i}, \mathrm{j}}^{\mathrm{H}} \mathbf{P} \mathbf{h}_{\mathrm{i}, \mathrm{j}}\right)
$$

Then:

$$
\begin{aligned}
& \mathrm{P}_{\mathrm{FA}}=\mathrm{Q}\left(\frac{\gamma-\mu_{\mathrm{T} \mid \mathrm{H}_{0}}}{\sigma_{\mathrm{T} \mid \mathrm{H}_{0}}}\right) \text { and } \mathrm{P}_{\mathrm{D}}=\mathrm{Q}\left(\frac{\gamma-\mu_{\mathrm{T} \mid \mathrm{H}_{1}}}{\sigma_{\mathrm{T} \mid \mathrm{H}_{1}}}\right) \\
& \mu_{\mathrm{T} \mid \mathrm{H}_{1}}=\sum_{\mathrm{i}=1}^{\mathrm{N}} \mathrm{a}_{\mathrm{i}} \mathrm{b}_{\mathrm{i}} \sum_{\mathrm{j}=1}^{\mathrm{N}_{\mathrm{f}}}\left\|\mathbf{P h}_{\mathrm{i}, \mathrm{j}}\right\|^{2} \\
& \sigma_{\mathrm{T} \mid \mathrm{H}_{1}}^{2}=\sum_{\mathrm{i}=1}^{\mathrm{N}} \mathrm{a}_{\mathrm{i}}^{2}\left[\mathrm{~b}_{\mathrm{i}}^{2} \sum_{\mathrm{j}=1}^{\mathrm{N}_{\mathrm{f}}} \sum_{\mathrm{k}=1}^{\mathrm{N}_{\mathrm{f}}}\left\|\mathbf{P h}_{\mathrm{i}, \mathrm{j}}\right\|^{2}\left\|\mathbf{P h}_{\mathrm{i}, \mathrm{k}}\right\|^{2}+\right. \\
& \sum_{\mathrm{j}=1}^{\mathrm{N}_{\mathrm{f}}}\left[\left\|\mathbf{P h}_{\mathrm{i}, \mathrm{j}}\right\|^{4}+\frac{\sigma^{2}}{2}\left\|\mathbf{P h}_{\mathrm{i}, \mathrm{j}}\right\|^{2}\right]-\left[\left[\mathrm{b}_{\mathrm{i}} \sum_{\mathrm{j}=1}^{\mathrm{N}_{\mathrm{f}}}\left\|\mathbf{P h}_{\mathrm{i}, \mathrm{j}}\right\|^{2}\right]^{2}\right]
\end{aligned}
$$

Here

$$
\mathrm{b}_{\mathrm{i}}=2 \mathrm{P}_{\mathrm{D}, \mathrm{i}}-1
$$

Similar computation for $\mu_{\mathrm{T} \mid \mathrm{H}_{1}}, \sigma_{\mathrm{T} \mid \mathrm{H}_{1}}^{2}$ wherein $\mathrm{b}_{\mathrm{i}}=2 \mathrm{P}_{\mathrm{D}, \mathrm{i}}-1$ replaced by $\mathrm{c}_{\mathrm{i}}=2 \mathrm{P}_{\mathrm{F}, \mathrm{i}}-1$.

Output signal power can be deduced from $\mathrm{S}=\sum_{\mathrm{j}=1}^{\mathrm{N}_{\mathrm{f}}} \operatorname{Re}\left(\mathbf{y}_{\mathrm{i}, \mathrm{j}}^{\mathrm{H}} \mathbf{P h _ { i }}\right)$, so the maximal SNR can be calculated as:

$$
\mathrm{SNR}=\frac{\sum_{\mathrm{j}=1}^{\mathrm{N}_{\mathrm{f}}} \operatorname{Re}\left(\mathbf{y}_{\mathrm{i}, \mathrm{j}}^{\mathrm{H}} \mathbf{P} \mathbf{h}_{\mathrm{i}, \mathrm{j}}\right)}{\sigma^{2}}
$$

\section{PROPOSAL}

From channel model [16], we can apply it to model between the SU and the FC. If changed as Fig. 2, considering the Angles of Arrival (AoAs): $\theta_{\mathrm{SUi}}, \mathrm{i}=1: \mathrm{N}$.

The channel model if using the beamforming is described in Fig.2. The received signal is below:

$$
\mathbf{y}_{\mathrm{i}}(\mathrm{l})=\mathbf{A}_{\mathrm{i}}(1) \mathbf{H}_{\mathrm{i}} \mathbf{u}_{\mathrm{i}}(1)+\mathbf{w}_{\mathrm{i}}(1)
$$

$\mathbf{A}_{\mathrm{i}}$ is matrix having size of $\mathrm{L}_{\mathrm{p}} \times \mathrm{N}_{\mathrm{f}}, \mathrm{L}_{\mathrm{p}}$ is number of paths form the ith SU to the FC. 
$\mathbf{u}_{\mathrm{i}}(1)$ is transmit signal from the $\mathrm{i}$ th $\mathrm{SU}$ at the time 1 , having size of $\mathrm{N}_{\mathrm{c}} \times 1$.

$\mathbf{H}_{\mathrm{i}}$ is channel matrix from the $\mathrm{i}$ th $\mathrm{SU}$ at the time 1 to the $\mathrm{FC}$, having size of $\mathrm{N}_{\mathrm{f}} \times \mathrm{N}_{\mathrm{c}}$.

$\mathbf{w}_{\mathrm{i}}(1)$ is noise vector received at the $\mathrm{FC}$, interfering the received signal $y_{i}(1)$, having size of $L_{p} \times 1$.

The received signal at the kth beam of the FC:

$$
\begin{aligned}
& \mathrm{y}_{\mathrm{i}, \mathrm{k}}(1)=\mathbf{a}_{\mathrm{i}, \mathrm{k}}(1) \mathbf{H}_{\mathrm{i}} \mathbf{u}_{\mathrm{i}}(1)+\mathrm{w}_{\mathrm{i}, \mathrm{k}}(1) \\
& \mathbf{y}_{\mathrm{i}}(1)=\left[\begin{array}{lllll}
\mathrm{y}_{\mathrm{i}, 1}(1) & \mathrm{y}_{\mathrm{i}, 2}(1) & . . & \mathrm{y}_{\mathrm{i}, \mathrm{L}_{\mathrm{p}}}(1)
\end{array}\right]^{\mathrm{H}} \\
& \mathbf{w}_{\mathrm{i}}(1)=\left[\begin{array}{lllll}
\mathrm{w}_{\mathrm{i}, 1}(1) & \mathrm{w}_{\mathrm{i}, 2}(1) & . . & \mathrm{w}_{\mathrm{i}, \mathrm{L}_{\mathrm{p}}}(1)
\end{array}\right]^{\mathrm{H}} \\
& \mathbf{H}_{\mathrm{i}}=\left[\begin{array}{lllll}
\mathbf{h}_{\mathrm{i}, 1} & \mathbf{h}_{\mathrm{i}, 2} & . . & \mathbf{h}_{\mathrm{i}, \mathrm{N}_{\mathrm{f}}}
\end{array}\right]^{\mathrm{H}} \text { where } \mathbf{h}_{\mathrm{i}, \mathrm{j}}^{\mathrm{H}} \text { has size of }
\end{aligned}
$$
$1 \times \mathrm{N}_{\mathrm{c}}$ therefore $\mathbf{H}_{\mathrm{i}}$ has size of $\mathrm{N}_{\mathrm{f}} \times \mathrm{N}_{\mathrm{c}}$.

$$
\mathbf{A}_{\mathrm{i}}(1)=\left[\begin{array}{llll}
\mathbf{a}_{\mathrm{i}, 1}(1) & \mathbf{a}_{\mathrm{i}, 2}(1) \quad \ldots \quad \mathbf{a}_{\mathrm{i}, \mathrm{L}_{\mathrm{p}}}(1)
\end{array}\right]^{\mathrm{H}}
$$

where $\mathbf{a}_{\mathrm{i}, \mathrm{k}}^{\mathrm{H}}(1), \mathrm{k}=1->\mathrm{L}_{\mathrm{p}}$ has size of $1 \times \mathrm{N}_{\mathrm{f}} . \quad \mathbf{A}_{\mathrm{i}}(1)$ has size of $L_{p} \times N_{f}$

$$
\mathbf{a}_{\mathrm{i}, \mathrm{k}}^{\mathrm{H}}(1)=\left[\begin{array}{c}
1 \\
\mathrm{e}^{-\frac{2 \pi}{\lambda} \sin \left(\theta_{i, \mathrm{k}}(1)\right)_{\mathrm{FU}}} \\
\cdots \\
\mathrm{e}^{-\frac{2 \pi}{\lambda}\left(N_{\mathrm{f}}-1\right) \sin \left(\theta_{i, \mathrm{k}}(1)\right)_{\mathrm{FU}}}
\end{array}\right], \mathrm{k}=1->\mathrm{L}_{\mathrm{p}}
$$

where $\mathrm{S}_{\mathrm{FU}}$ is the antenna spacing of the $\mathrm{FC}$.

If we combine the $\mathrm{L}$ transmit vectors ( $\mathrm{L}$ beams), then we have the signal at the $\mathrm{k}$ th beam of the FC:

$$
\mathbf{y}_{\mathrm{i}, \mathrm{k}}=\mathbf{U}_{\mathrm{i}} \mathbf{H}_{\mathrm{i}}^{\mathrm{H}} \mathbf{a}_{\mathrm{i}, \mathrm{k}}+\mathbf{w}_{\mathrm{i}, \mathrm{k}}
$$

where

$$
\begin{aligned}
& \mathbf{y}_{\mathrm{i}, \mathrm{k}}=\left[\begin{array}{llll}
\mathrm{y}_{\mathrm{i}, \mathrm{k}}(1) & \mathrm{y}_{\mathrm{i}, \mathrm{k}}(2) & \ldots & \mathrm{y}_{\mathrm{i}, \mathrm{k}}(\mathrm{L})
\end{array}\right]^{\mathrm{H}} \\
& \mathbf{w}_{\mathrm{i}, \mathrm{k}}=\left[\begin{array}{llll}
\mathrm{w}_{\mathrm{i}, \mathrm{k}}(1) & \mathrm{w}_{\mathrm{i}, \mathrm{k}}(2) & . . & \mathrm{w}_{\mathrm{i}, \mathrm{k}}(\mathrm{L})
\end{array}\right]^{\mathrm{H}} \\
& \mathbf{a}_{\mathrm{i}, \mathrm{k}}=\mathbf{a}_{\mathrm{i}, \mathrm{k}}(1)=\left[\begin{array}{llll}
\mathrm{a}_{\mathrm{i}, \mathrm{k}}(1) & \mathrm{a}_{\mathrm{i}, \mathrm{k}}(2) & . . & \mathrm{a}_{\mathrm{i}, \mathrm{k}}\left(\mathrm{N}_{\mathrm{f}}\right)
\end{array}\right]^{\mathrm{H}} \\
& \mathbf{U}_{\mathrm{i}}=\left[\begin{array}{llll}
\mathbf{u}_{\mathrm{i}}(1) & \mathbf{u}_{\mathrm{i}}(2) & . . & \mathbf{u}_{\mathrm{i}}(\mathrm{L})
\end{array}\right]^{\mathrm{H}} \text { has size of } \mathrm{L} \times \mathrm{N}_{\mathrm{c}}
\end{aligned}
$$

From this, if we are keeping (10), we have changed (11) as below:

$$
\begin{aligned}
& \mathrm{P}_{\mathrm{FA}}=\mathrm{Q}\left(\frac{\gamma-\mu_{\mathrm{T} \mid \mathrm{H}_{0}}}{\sigma_{\mathrm{T} \mid \mathrm{H}_{0}}}\right) ; \mathrm{P}_{\mathrm{D}}=\mathrm{Q}\left(\frac{\gamma-\mu_{\mathrm{T} \mid \mathrm{H}_{1}}}{\sigma_{\mathrm{T} \mid \mathrm{H}_{1}}}\right) \\
& \mu_{T \mid H_{1}}=\sum_{i=1}^{N} a_{i} b_{i} \sum_{j=1}^{\mathrm{L}}\left\|\mathbf{P} \mathbf{H}_{i}^{H} \mathbf{a}_{i, j}\right\|^{2} \\
& \sigma_{\mathrm{T} \mid \mathrm{H}_{\mathrm{l}}}^{2}=\sum_{\mathrm{i}=1}^{\mathrm{N}} \mathbf{a}_{\mathrm{i}}^{2}\left[\mathrm{~b}_{\mathrm{i}}^{2} \sum_{\substack{\mathrm{j}=1 \\
\mathrm{~L}_{\mathrm{p}}} \sum_{\mathrm{k}=1}^{\mathrm{L}_{\mathrm{p}}} \| \mathbf{j}}\left\|\mathbf{P H}_{\mathrm{i}}^{\mathrm{H}} \mathbf{a}_{\mathrm{i}, \mathrm{j}}\right\|^{2}\left\|\mathbf{P H}_{\mathrm{i}}^{\mathrm{H}} \mathbf{a}_{\mathrm{i}, \mathrm{k}}\right\|^{2}\right] \\
& +\sum_{\mathrm{i}=1}^{\mathrm{N}} \mathbf{a}_{\mathrm{i}}^{2}\left[\sum_{\mathrm{j}=1}^{\mathrm{L}_{\mathrm{p}}}\left[\left\|\mathbf{P H}_{\mathrm{i}}^{\mathrm{H}} \mathbf{a}_{\mathrm{i}, \mathrm{j}}\right\|^{4}+\frac{\sigma^{2}}{2}\left\|\mathbf{P} \mathbf{H}_{\mathrm{i}}^{\mathrm{H}} \mathbf{a}_{\mathrm{i}, \mathrm{j}}\right\|^{2}\right]\right] \\
& -\sum_{i=1}^{N} a_{i}^{2}\left[\left[b_{i} \sum_{j=1}^{L_{p}}\left\|\mathbf{P H}_{i}^{H} \mathbf{a}_{i, j}\right\|^{2}\right]^{2}\right]
\end{aligned}
$$

Herein

$$
\mathrm{b}_{\mathrm{i}}=2 \mathrm{P}_{\mathrm{D}, \mathrm{i}}-1
$$

Now, the signal to interference ratio is:

$$
\begin{gathered}
\operatorname{SNR}_{\mathrm{BF}}=\frac{\sum_{\mathrm{k}=1}^{\mathrm{L}_{\mathrm{p}}} \operatorname{Re}\left(\mathbf{y}_{\mathrm{i}, \mathrm{k}}^{\mathrm{H}} \mathbf{P} \mathbf{H}_{\mathrm{i}}^{\mathrm{H}} \mathbf{a}_{\mathrm{i}, \mathrm{k}}\right)}{\sigma^{2}} \\
=\frac{\sum_{\mathrm{k}=1}^{\mathrm{L}_{\mathrm{p}}} \operatorname{Re}\left(\left(\mathbf{U}_{\mathrm{i}} \mathbf{H}_{\mathrm{i}}^{\mathrm{H}} \mathbf{a}_{\mathrm{i}, \mathrm{k}}+\mathbf{w}_{\mathrm{i}, \mathrm{k}}\right)^{\mathrm{H}} \mathbf{P} \mathbf{H}_{\mathrm{i}}^{\mathrm{H}} \mathbf{a}_{\mathrm{i}, \mathrm{k}}\right)}{\sigma^{2}}
\end{gathered}
$$

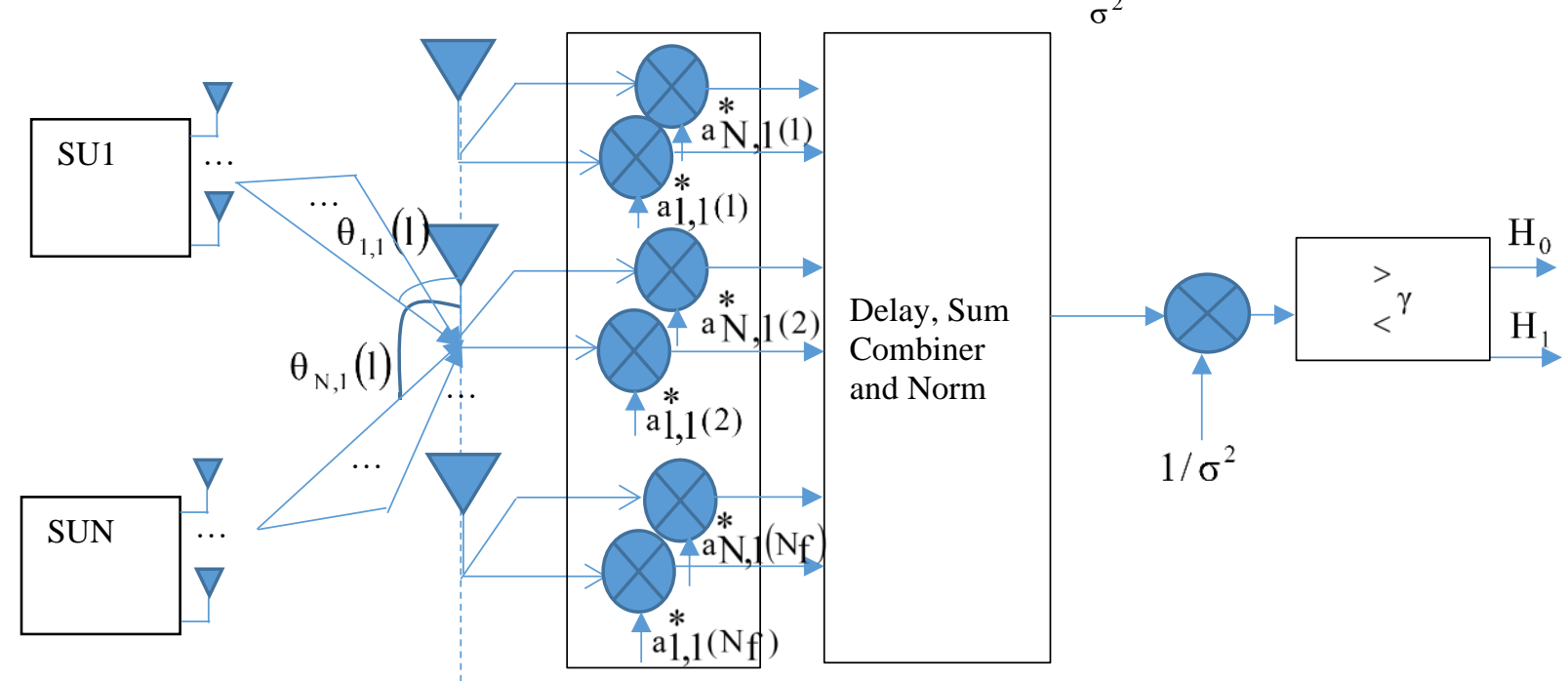

Fig. 2. Proposed beamforming for path 1 to all SUs at the FC. 


\section{SIMULATION}

We can use the hierarchical codebook (HC) for improving the detection performance at the FC. In essence, the HC pairing of the transmit and receive beams to optimize the channel rate between the SUs and the FC. In other words, the optimal pair of the transmit and receive beams will help to increase the signal power to noise ratio SNR at the FC. This increases the FC's ability to detect the PU. We can use simulation to demonstrate the advantage of using the transmit and receive beams to improve the detection quality of the PU at the FC. A typical hierarchical codebook is illustrated in Fig. 3.

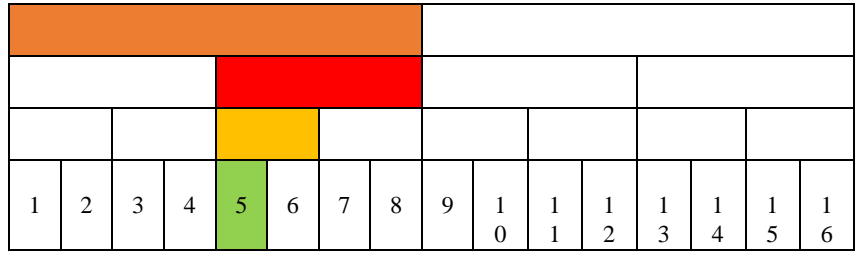

Fig. 3. Codebook 4 layers (16 beams at the last layer) with hierarchical searching for the optimum beam.

We can divide into the cases:

1. Using a codebook consisting of two codewords, each codeword will form a beam, described as shown in Fig. 4.

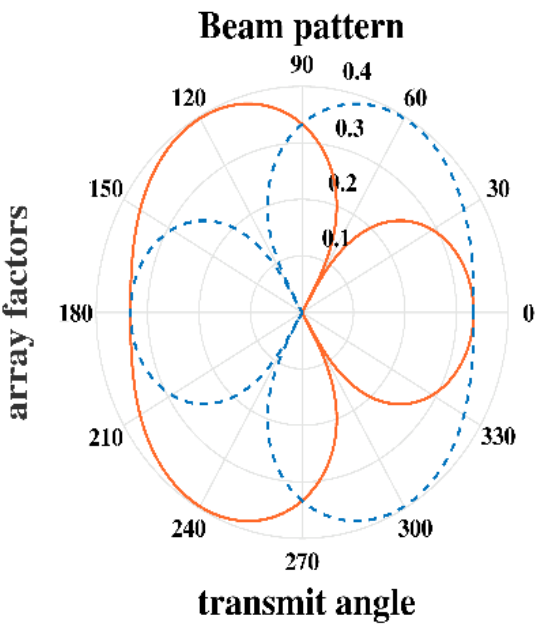

Fig. 4. Using two beams of the codebook.

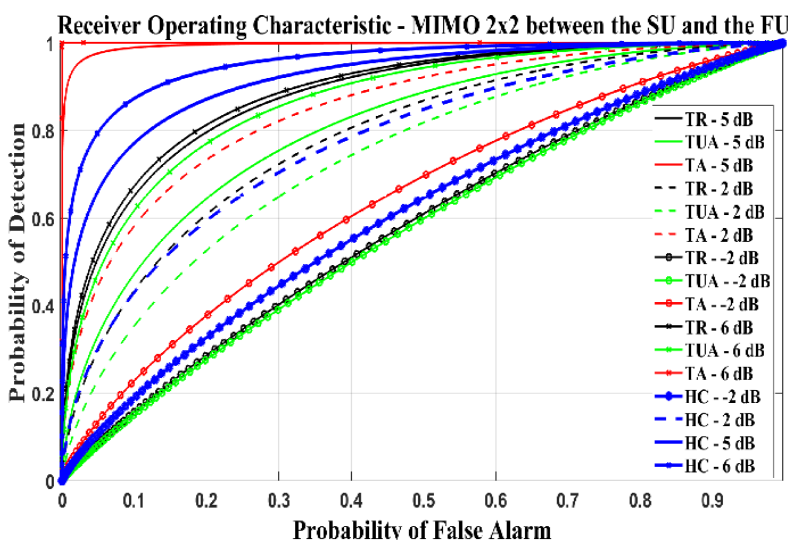

Fig.5. Comparison between using HC and other methods in case of different SNR in MIMO $2 \times 2$
When we use SNR with corresponding values of $-2 \mathrm{~dB}, 2$ $\mathrm{dB}, 5 \mathrm{~dB}$, and $6 \mathrm{~dB}$, we find that in Fig. 5 that is applying $\mathrm{HC}$ 2 beams is better than Robust Test (TR), concerning the covariance matrix, the channel estimates known at the FC and Uncertainty Agnostic Test (TUA), relating to only estimates of channel matrix available at the FC [15]

2 . Using a codebook of 16 codewords, each codeword will form a beam, described in Fig. 6.

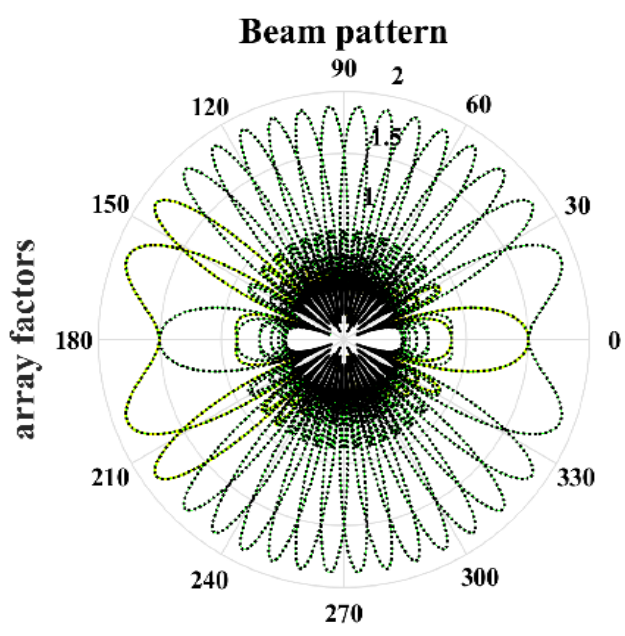

transmit angle

Fig. 6. Using 16 beams of the codebook.

When we use 16 beams, it becomes easier to detect the presence of the PU because the probability of detection is high even when the probability of false alarm is small. We compare the four methods, and we can see that in addition to the method using the TA when the channel state information is perfectly known at the $\mathrm{FC}$, the $\mathrm{HC}$ is still better than the two methods using the TR using channel state statistics (channel correlation matrix) and the TUA method when the FC knows the nominal channel state information, illustrated in Fig.7. Here we simulate with only $\mathrm{SNR}=-2 \mathrm{~dB}$. The TA is the best solution as this case assumes that the channel matrix is perfectly known at the FC. The HC is the proposed solution when we consider the beam pair for the SUs and the FC first that makes the SNR higher at the FC, then show these beams make the detection and the fault alarm probabilities more improved than the TR and the TUA. Of course, the parameter for the $\mathrm{HC}$ is still lower the ideal case, in which the TA is utilized at the FC.

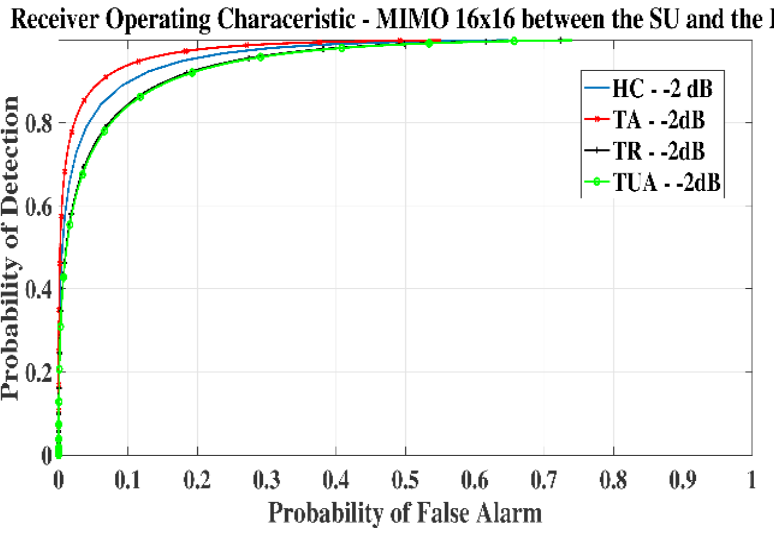

Fig. 7. Comparison between using $\mathrm{HC}$ and other methods for $\mathrm{SNR}=-2 \mathrm{~dB}$ in MIMO $16 \times 16$ 
3. If I use a codebook of 64 codewords, the number of 64 beams is shown in Fig. 8 .

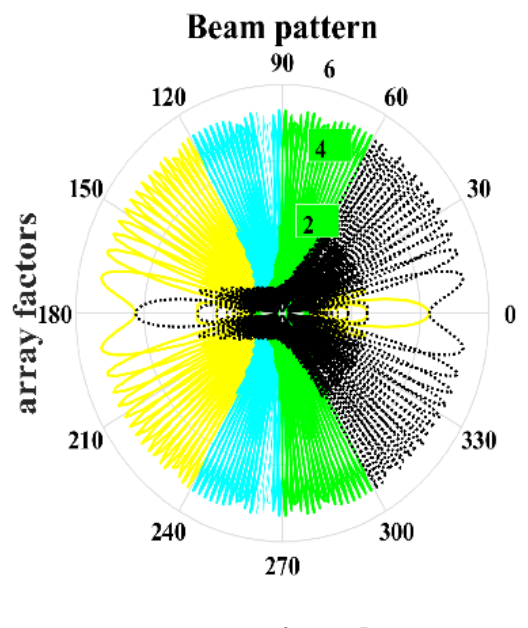

transmit angle

Fig. 8. Using 64 beams of the codebook.

If we use 64 beams, the TA, TR and TUA systems as well as the HC system achieve a higher detection probability than in the case of 2 beams or 16 beams. Here, the method using $\mathrm{HC}$ still gives a higher detection probability than the two cases TR and TUA, illustrated in Fig. 9.

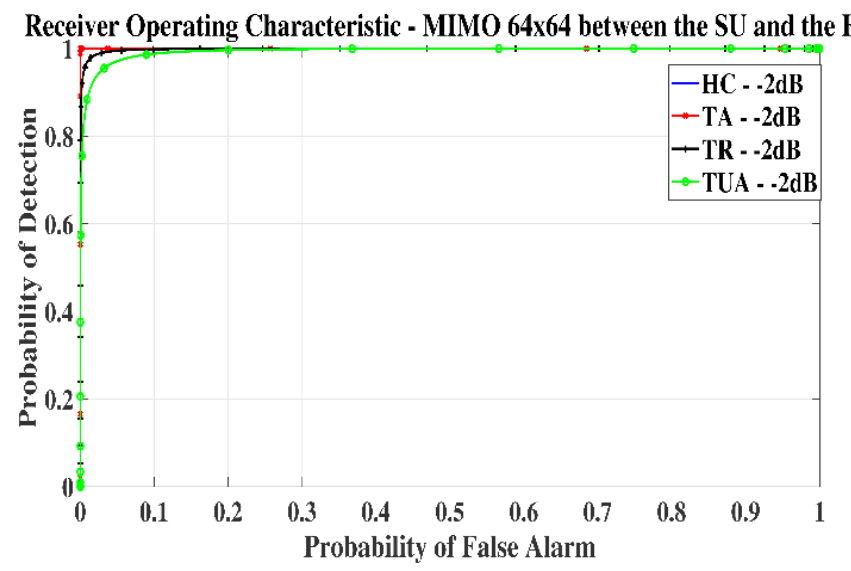

Fig. 9. Comparison between using $\mathrm{HC}$ and other methods for $\mathrm{SNR}=-2 \mathrm{~dB}$ in MIMO $64 \times 64$.

\section{CONCLUSION}

Thus, we have converted the discovery of the presence of the PU from the traditional MIMO model to the MIMO model with additional beamforming techniques combined with the hierarchical codebook. Using the beamforming technique helps the receiver (FU) have more chances to find out the existence of the PU. This is because the SNR at the FU is higher than the traditional MIMO model. With this proposed model and the different types of hierarchical codebooks, the detection probability increases rapidly compared to the traditional model, namely the method using channel matrix correlation (for the UA) and nominal matrix estimates (for the TUA) at the FC.

\section{ACKNOWLEDGMENT}

I thank the University of Transport and Communications for support to this paper.

\section{REFERENCES}

[1] Abhishek Kumar, Tripta, Seemanti Saha. A decision confidence based multiuser MIMO cooperative spectrum sensing in CRNs. Physical Communication 2020; https://doi.org/10.1016/j.phycom2019.100995.

[2] Nan Zhao, Fei Richard Yu, Hongjian Sun and Arumugam Nallanathan Energy-efficient cooperative spectrum sensing schemes for cognitive radio networks. EURASIP Journal on Wireless Communications and Networking 2013; 2013:120. https://doi.org/10.1186/1687-1499-2013120 .

[3] Lu Lv; Jian Chen; Qiang Ni; Zhiguo Ding; Hai Jiang. Cognitive nonorthogonal multiple access with cooperative relaying: a new wireless frontier for $5 \mathrm{G}$ spectrum sharing. IEEE Communications Magazine 2018; 56(4): 188-195. 10.1109/MCOM.2018.1700687.

[4] Mehdi Ghamari Adian. Beamforming with reduced complexity in MIMO cooperative cognitive radio networks. Hindawi Publishing Corporation 2014. http://dx.doi.org/10.1155/2014/325217.

[5] Shakeel A. Alvi, Riaz Hussain, Atif Shakeel, Muhammad Awais Javed, Qadeer Ul Hasan, Byung Moo Lee, and Shahzad A. Malik. QoSoriented optimal relay selection in cognitive radio networks. Hindawi Publishing Corporation 2021. https://doi.org/10.1155/2021/5580963.

[6] Son Dinh, Hang Liu, Feng Ouyang, Massive MIMO cognitive cooperative relaying, springer LNCS wireless algorithms. Systems, and Applications 2019; 11604: 98-110. https://doi.org/10.1007/978-3-03023597-0_8.

[7] Ghamari Adian Mehdi, Aghaeinia Hassan. Joint relay selection and power allocation in MIMO cooperative cognitive radio networks Journal Of Information Systems and Telecommunication (JIST) 2015; 3:1(9): 29-40.

[8] Advaita Advaita, Mani Meghala Gali, Thi My Chinh Chu, and HansJurgen Zepernick. Outage Probability of MIMO Cognitive Cooperative Radio Networks with Multiple AF Relays Using Orthogonal SpaceTime Block Codes. IEEE 13th International Conference on Wireless and Mobile Computing, Networking and Communications (WiMob) 2017. 10.1109/WiMOB.2017.8115749.

[9] Wei Chen, Liang Hong. Cooperative MIMO paradigms for cognitive radio networks. IEEE International Symposium on Parallel \& Distributed Processing, Workshops and Phd Forum. 2013. 10.1109/IPDPSW.2013.9.

[10] Amr Y. Elnakeeb; Hany M. Elsayed; Mohamed M. Khairy. Clustering for cooperative MIMO cognitive radio sensor networks under interference constraints. 9th International Conference on Cognitive Radio Oriented Wireless Networks and Communications (CROWNCOM) 2014. 10

[11] Gangtao Han, Jian-Kang Zhang, Xiaomin Mu and Xinying Guo. MIMO cooperative cognitive radio relay networks with uniquelyfactorable constellation pair. IEEE 17th International Workshop on Signal Processing Advances in Wireless Communications (SPAWC) 2016. 10.1109/SPAWC.2016.7536883.

[12] Waleed Ejaz, Ghalib A. Shah, Najam ul Hasan and Hyung Seok Kim. Energy and throughput efficient cooperative spectrum sensing in cognitive radio sensor networks. Trans. Emerging Tel. Tech 2014 DOI: $10.1002 /$ ett.2803.

[13] S Hariharan, S Venkata Siva Prasad and P Muthuchidambaranathan Average detection probability analysis for cooperative - MIMO spectrum sensing in cognitive radio networks. International Conference on Advances in Computing, Communications and Informatics (ICACCI) 2013. 10.1109/ICACCI.2013.6637159.

[14] Muthana Al-Amidie, Ahmed Al-Asadi, Amjad J. Humaidi , Ayad AlDujaili , Laith Alzubaidi, Laith Farhan, Mohammed A. Fadhel, Ronald G. McGarvey and Naz E. Islam. Robust spectrum sensing detector based on MIMO cognitive radios with non-perfect channel gain. Electronics 2021, 10, 529. https://doi.org/10.3390/electronics 10050529.

[15] Adarsh Patel, Hukma Ram, Aditya K. Jagannatham, and Pramod K. Varshney, Robust cooperative spectrum sensing for MIMO cognitive radio networks under CSI uncertainty. IEEE Transactions on Signal Processing 2018; 66(1): 18-33. 10.1109/TSP.2017.2759084.

[16] Amr Hussein Hussein, Hager Shawky Fouda, Haythem Hussein Abdullah and Ashraf A. M. Khalaf. A highly efficient spectrum sensing approach based on antenna arrays beamforming. IEEE Access 2020; 8: 25184 - 25197. 10.1109/ACCESS.2020.2969778. 
[17] Zhenyu Xiao, Tong He, Pengfei Xia and Xiang-Gen Xia, Hierarchical codebook design for beamforming training in millimeter-wave communication, IEEE Transactions on Wireless Communications 2016, 15(5): 3380-3392. 10.1109/TWC.2016.2520930.

[18] Hoai Trung Tran. Using effective codebook in hybrid precoding for MIMO mm-wave communication. International Journal of Microwave and Optical Technology 2020; 15(4): 325-334.

[19] Hoai Trung Tran. Doppler frequency offset compensation using hierarchical codebook at the moving receiver. International Journal of Microwave And Optical Technology 2021; 16(5): 459-469.

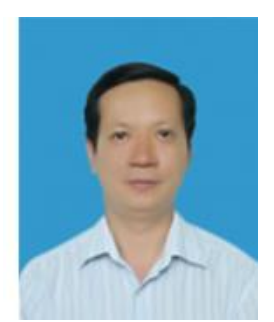

Hoai Trung Tran was born in 1976. He got Bachelor degree in University of Transport and Communications (UTC) in 1997 and hold the post of lecturer at the University. He then got a Master degree from Hanoi University of Science and Technology (HUST) in 2000. In the period 2003 to 2008 , he had concentrated on researching in the field of Telecommunication engineering and got his $\mathrm{PhD}$ at University of Technology, Sydney (UTS) in Australia. He is currently lecturer at the UTC. His main research interests are digital signal processing (DSP), applied information theory, radio propagation, MIMO antenna techniques and advanced wireless transceiver design. 